\title{
Welcome to ICN 2010
}

\author{
Herbert Budka
}

Published online: 20 July 2010

(C) Springer-Verlag 2010

As the President of the Organizing Committee of the XVIIth International Congress of Neuropathology (ICN 2010), I am delighted to be given the opportunity to introduce this issue of ACTA NEUROPATHOLOGICA, to be distributed to participants of ICN 2010. In addition to the book of abstracts, to be printed in a supplementary issue of BRAIN PATHOLOGY, the present series of review articles illustrates well the meeting and its scope. An ICN should be dear to all neuropathologists, as this global event brings together the research and medical-diagnostic aspects of the field in one place only every 4 years. Originally, we expected to attract an attendance of up to 800-1,000 researchers, medical specialists and industry representatives; however, the global crisis seems to have a more profound impact on the number of registrants than we anticipated. This hurts in particular, as we decided to design ICN 2010 as a low-budget, non-profit event, in order to keep costs for attendees as affordable as possible, in particular for young scientists, students and trainees and those from economically disadvantaged countries. Fortunately, however, there is no impact on the quality of scientific presentations and discussions, as also testified by the contributions to this issue of ACTA NEUROPATHOLOGICA.

The scientific program of ICN 2010 covers topics that span the whole range of areas of interest for neuropathologists: there is no other event for them that compares with the breadth and depth of information to be gained there. With regard to topics of the submitted abstracts, neurodegeneration and tumours predominate and equal each other, with

H. Budka $(\bowtie)$

Institute of Neurology, Medical University of Vienna, AKH 4 J, Währinger Gürtel 18-20, 1097 Vienna, Austria e-mail: herbert.budka@meduniwien.ac.at one-third each. While this is hardly surprising, it may become a matter of concern if neuropathology lessens, or even loses, its grip on the full spectrum of neuropsychiatric diseases that have been in its domain since decades. ICN 2010 tries to counter-balance such trends: while these topics do get the attention they rightly deserve, it is not a neurodegeneration- and tumour-only meeting. In particular, invited speakers from neighbouring disciplines provide insight that is difficult if not impossible for neuropathologists to get elsewhere in comparable comprehensiveness. Some of these exemplary invited lectures are published in this journal issue: V. Hugh Perry discusses the contribution of systemic inflammation to neurodegeneration-a major source of orientation in a turf where (neuro)immunologists and neurodegeneration researchers meet-and also come apart. A similar dualistic situation applies to neurodegeneration and cerebrovascular disease; Costantino Iadecola analyses the overlap between neurodegenerative and vascular factors in the pathogenesis of dementia. Problems of tumour neuropathology are discussed by Martin van den Bent from a clinician's perspective, while David Ellison provides data for new classifications of medulloblastoma. In the area of infectious and inflammatory disorders that is of pivotal importance in many parts of the globe, Kum Thong Wong focuses on epidemic viral encephalitis, in particular by the emerging henipaviruses. The group of Tamas Revesz covers the role of brain biopsy in dementia, an issue that reemerges with progress in fine-tuning of neuropathological diagnoses (and possibly also with decline of autopsy rates). Muscle pathology has to remain an intrinsic part of neuropathology; Caroline Sewry updates on the rapid progress in muscular dystrophies that demonstrates how difficult it has become for a neuropathology laboratory to provide state-of-art diagnostic service. With increasing and widespread use of anti-epileptic surgery, there is a need for neuropathologists 
to keep pace; Roberto Spreafico and Ingmar Blümcke present a new neuropathological classification of focal cortical dysplasia. Intriguing implications for pathogenesis of Alzheimer disease have emerged from studies of the neuropathology after $\mathrm{AD}$ vaccination, as demonstrated by the group of James Nicoll. Finally, William Hu and John Trojanowski give perspectives for the future development of neuropathology, with emphasis on biomarkers for neurodegenerative diseases. However, these interesting articles are only some examples why ICN 2010 in Salzburg will be spectacular: participants can enjoy top-class science in a cosy and relaxed atmosphere and in a unique cultural setting.

Having now ICN 2010 as the most recent display of global neuropathology in science and medical service, is it possible to foresee where the discipline will stand in 15 or 20 years? Clearly, simple morphology has become, and even more will become, less and less relevant, to be replaced by sophisticated molecular methodology, biobanking and shifting to biomarkers; if neuropathology is able to adapt, its future is far from endangered. It is up to us and to our young trainees to take up this challenge: " $L a$ chance sourit à l'esprit prépare" (Louis Pasteur).

WELCOME TO SALZBURG AND ICN 2010!

Herbert Budka

President ICN 2010 